Classification

Physics Abstracts

02.81 - 07.05.Pj - 07.05.Rm - 07.60.-j - 42.30-68.35.Bs

\title{
Study of the Contact Permeability between Rough Surfaces from Confocal Microscopy
}

\author{
Claire-Hélène Demarty $\left({ }^{1}\right)$, François Grillon $\left({ }^{2}\right)$ and Dominique Jeulin $\left({ }^{1}\right)$ \\ $\left({ }^{1}\right)$ Centre de Morphologie Mathématique, École des Mines de Paris, 35 rue Saint-Honoré, \\ 77305 Fontainebleau, France \\ $\left({ }^{2}\right)$ Centre des Matériaux Pierre Marie Fourt, École des Mines de Paris, BP 87, 91003 Evry Cedex, \\ France
}

Résumé. - Dans cet article, nous étudions un processus complet de mise en contact et de mesure de perméabilité de surfaces rugueuses. Les images, acquises par microscopie confocale, sont ensuite traitées par des traitements d'images 3-D. Nous donnons tout d'abord deux techniques de correction de la dérive introduite dans les images lors de l'acquisition. Puis nous décrivons les algorithmes de mise en contact de deux surfaces rugueuses l'une contre l'autre et de propagation 3-D d'un fluide entre les deux. Nous présentons enfin les résultats obtenus sur des images de joints céramiques, sous la forme de chemin et temps minimaux de propagation.

\begin{abstract}
In this paper, we present a study of the contact permeability between rough surfaces by means of 3-D image processing. Three-dimensional images of the surfaces were acquired from confocal microscopy. We first give two methods of image processing in order to correct the drift introduced in images through confocal microscopy. Algorithms to bring surfaces into contact and 3-D propagation between the surfaces are then described. The results obtained by experiments on ceramic joints, under the form of minimal path and minimal time of propagation in the surfaces, are also presented.
\end{abstract}

\section{Introduction}

The aim of this study is to get information on the contact permeability between two rough surfaces. In this paper, the surfaces come from ceramic joints for taps. This study is part of a project contracted by the Ceramiques Techniques Desmarquest [3], in order to determine the percolation threshold of two tap joints in contact. Thanks to confocal microscopy, digital elevation maps of each surface are acquired. The acquisition is then followed by three steps of 3-D image processing: first a drift correction of the images, then the bringing into contact of two surfaces, and the simulation of the 3-D propagation. 


\section{Data}

The data are obtained by using a TSM NORAN microscope. A 100LWD objective was chosen, which gives a scale of $0.25 \mu \mathrm{m}$ in the $x$ and $y$ directions, for $512 \times 512$ images. A short estimation of the maximal difference in height is about $10 \mu \mathrm{m}$. In order to retain the isotropy in the three directions, the scale of $z$ is $0.22 \mu \mathrm{m}$. Each acquisition is then constituted of 45 slices. This sampling condition was also validated by a study of the Euler number according to different numbers of slices.

To each slice corresponds one grey level. Therefore, each digital elevation map represents a 3 -D volume of $130 \times 130 \times 10 \mu \mathrm{m}^{3}$, under the form of a $2 \mathrm{D}$ image with 45 gray levels.

\section{Drift Correction}

During the confocal microscope acquisition and because of optical defects, the images of the surfaces present a slight drift, which must be removed, in order to bring both surfaces into contact. To do so, one indeed needs to have perfectly parallel samples, not to obtain false permeability measurements. Two different methods of drift correction were developed, according to the type of samples.

The main tool used to correct the drift is an image processing operator: the closing by reconstruction. This operator is derived from the theory of mathematical morphology, which proposes a powerful toolbox of non linear image processing operators. Based on algebra and set theory, it studies object shapes in an image, according to elementary shapes named structuring elements. The closing by reconstruction or mathematical morphology will not be described in this paper, but one can refer to $[12,11,10]$ for further explanations and definitions.

Applying the closing by reconstruction to a joint image, one is able to fill the image holes and to keep all the hills and the flat zones unchanged. In the case of well-polished samples, flat zones prevail and give the average drift of the image. A simple subtraction reconstructs the image without any drift (Fig. 1, top line: the original image is on the left and the corrected image on the right).

For well-polished samples, the correction is almost perfect. However in the case of roughly polished samples (specially prepared for this study), for which the image still contains numerous maxima, the subtraction of the closing by reconstruction makes them disappear. For this type of samples, the relief is modified by the correction. We therefore need another technique of correction.

As the drift looks roughly like a plane for the chosen objective, the second method consists in an estimation of the best plane which describes the sample surface. The plane parameters are optimized, by mean square error minimization. After subtraction of the estimated plane, the corrected image is obtained, without modifying the relief. This is illustrated in Figure 1, in the bottom line.

One or the other method (the closing by reconstruction or the plane estimation) is chosen according to the sample roughness.

Figure 2 gives two different height distributions. These histograms correspond respectively to a "peak and hole" structure for the Gaussian histogram and to a "flat zone" structure for the other. This illustrates the two types of joint structures: roughly polished (Gaussian) and well-polished (distribution with a sharp peak). 


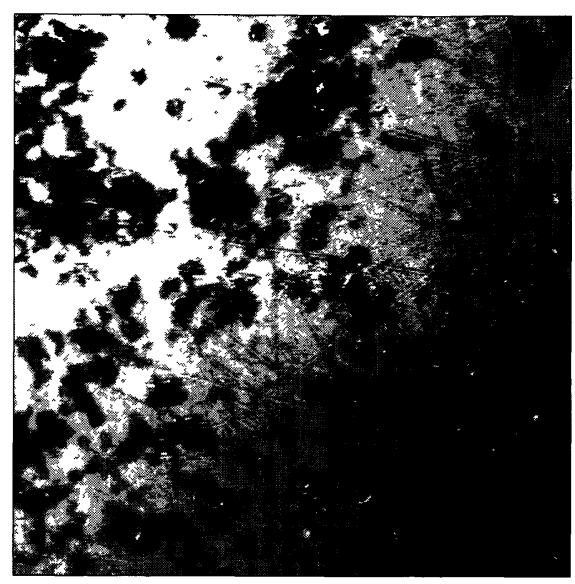

Original image

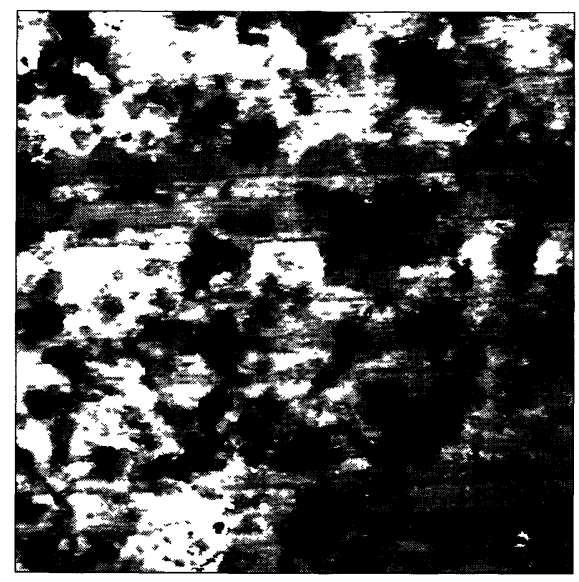

Original image

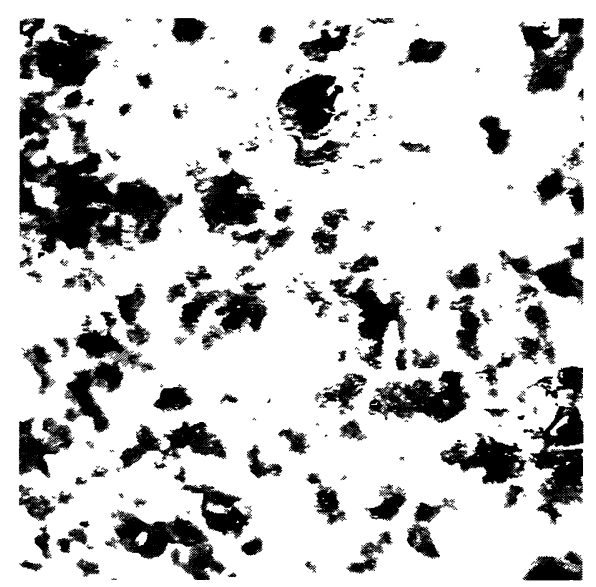

Corrected image

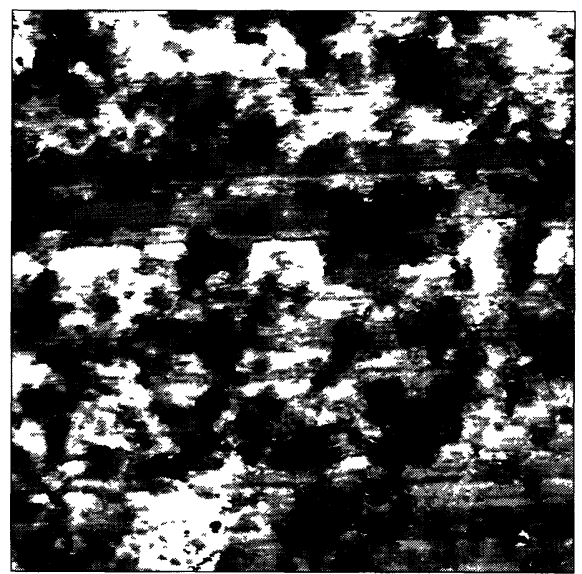

Corrected image

Fig. 1. - Example of drift correction (top line: closing by reconstruction, well-polished sample, bottom line: plane estimation, roughly polished sample). $\left(130 \times 130 \times 10 \mu \mathrm{m}^{3}\right)$.

\section{Simulation of the Contact between Two Specimens}

After the acquisition and the drift correction of each image, the contact between two samples should be simulated, in order to reconstruct the volume of the interval between two joints.

The contact algorithm should answer the following question: what is the best position of one joint over another in terms of minimal volume of the pores and maximal vertical fit? However during real contact, the two joints take the first stable equilibrium position and the two above criteria are not always satisfied. To simulate as well as possible the reality, the algorithm starts from two joints one above the other and searches the best fit according to translations limited to a small window. Once this starting position is found, and if it corresponds to one edge of the window, small horizontal translations are allowed in order to obtain a better fit outside the window. But once again, if a stable position is found, the contact process stops. This algorithm therefore satisfies the maximal vertical fit, but on a small window only. 


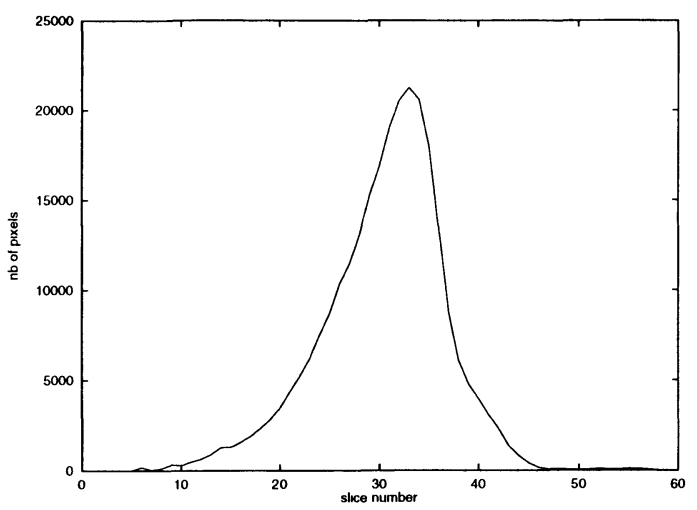

a)

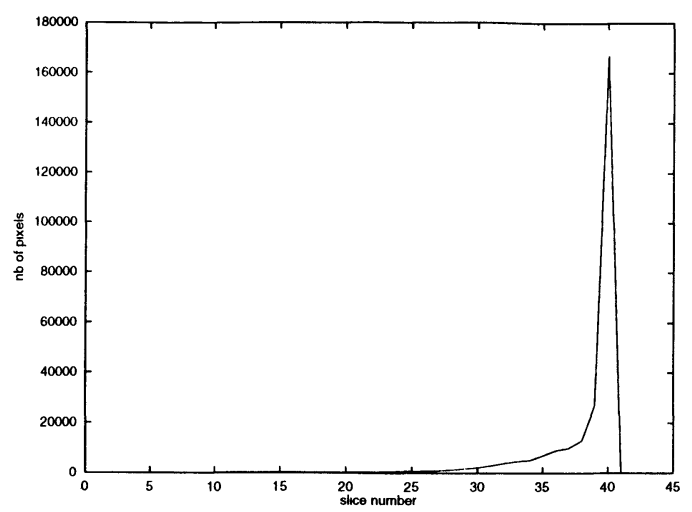

b)

Fig. 2. - Height distributions. a) Roughly polished joint. b) Well-polished joint.

\section{3-D Propagation}

The three-dimensional propagation is similar to the simulation of a fluid propagation in a porous medium. Fluid propagation in porous media, and geodesic distance, which is the basis of propagation, were already studied in the case of two dimensional media $[7,8,1,6]$. The propagation makes the calculation of a geodesic distance from a given set of voxels, namely the source of the propagation to every voxel of the 3-D sample. In the calculation, use is made of a graph built on the cubic grid (every voxel having 6 nearest neighbours). The access to voxels located inside the rough surface is forbidden. This approach can be used to estimate a percolation threshold from simulations, as made in 3-D [9]. The used algorithm presents the advantage of being based on hierarchical queues; furthermore it can be applied to general 3-D domains [5, 4, 2].

Because of the three-dimensional volumes to process $(512 \times 512 \times 90$ images $)$, the algorithm is memory consuming. It is possible to reduce its memory requirements, since the interesting part of the joint volume is reduced to the "in between part", between the two surfaces. This part could be accessed through a surface following algorithm.

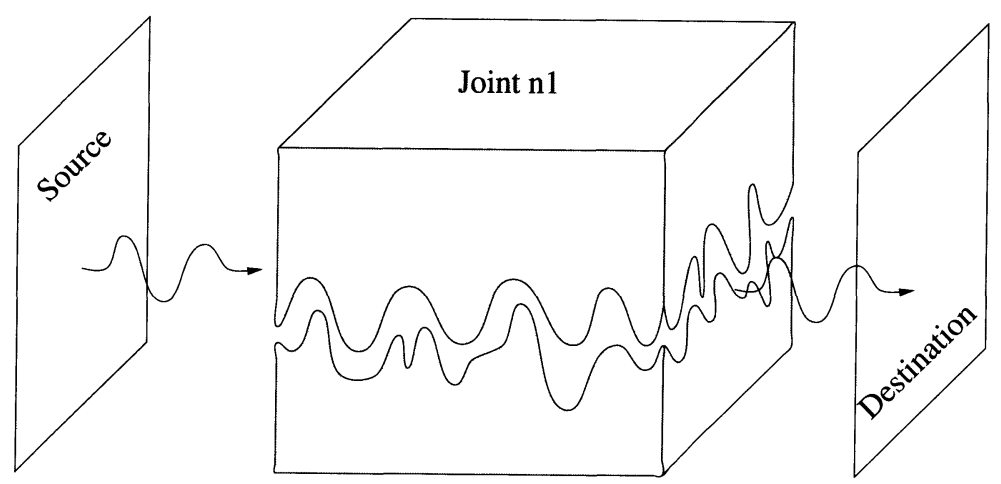

Joint n2

Fig. 3. - Source and destination planes. 


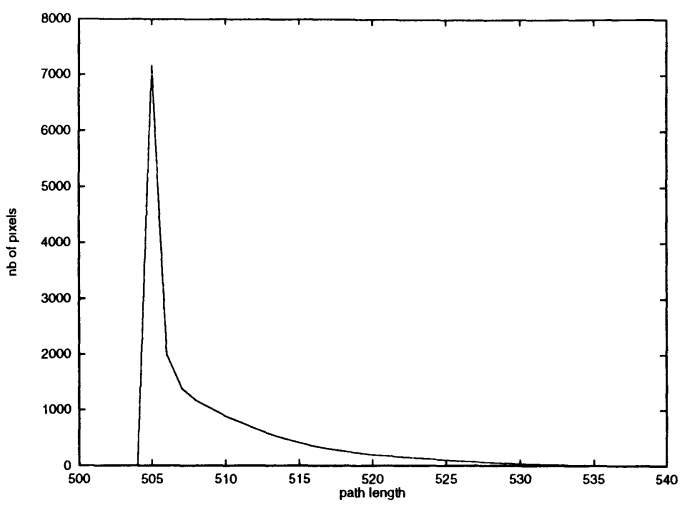

a)

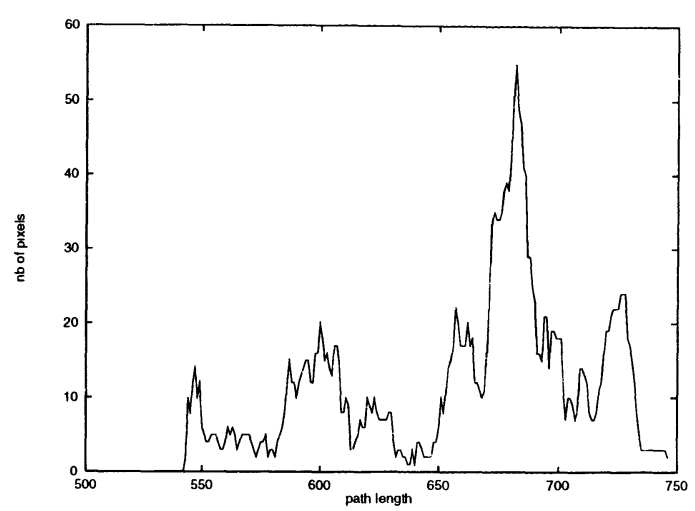

b)

Fig. 4. - Distribution of path lengths for the destination plane (porous samples: a) roughly polished, b) well-polished).

a)

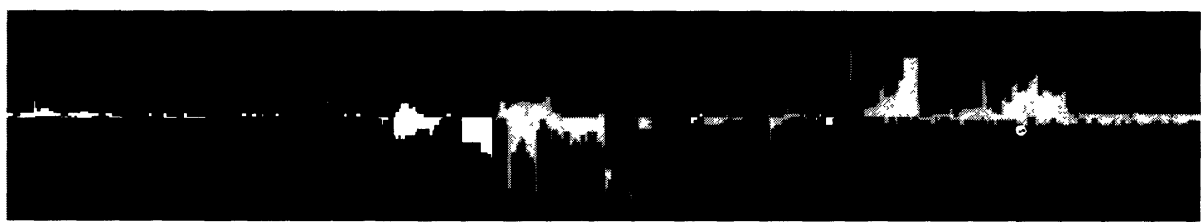

b)

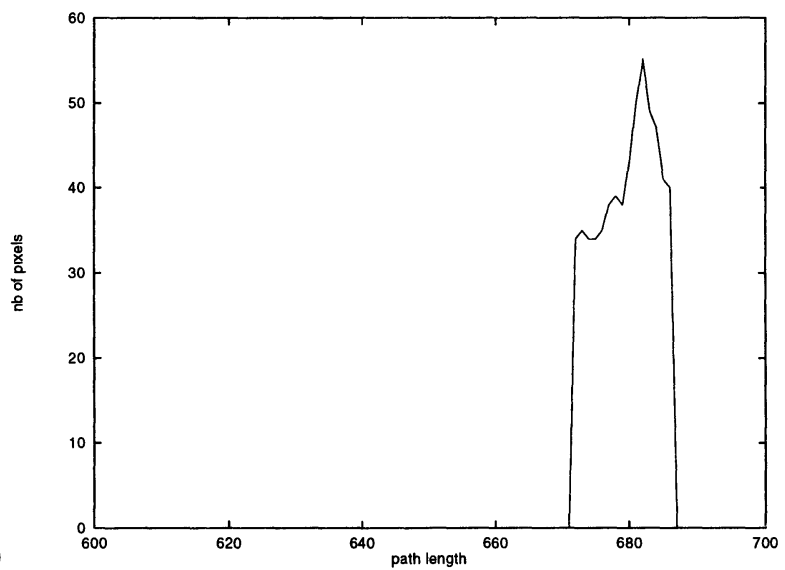

Fig. 5. - Shortest path cartography for the destination plane. a) Path cartography for the destination plane. In white, pores unreached by the propagation; in dark gray, pores belonging to paths of more than 20 pixels in the destination plane; in light gray, the rest of the reached pores. b) Histogram threshold for the paths of more than 20 pixels.

However a more general algorithm for 3-D domains was used. For our samples, the propagation is made in $15 \mathrm{mn}$ on a Sun Sparc 10 for a $512 \times 512 \times 90$ image and most of the time is taken by memory swapping of the $3-\mathrm{D}$ image. This could be reduced with more RAM (>128 Mo). Furthermore only $10 \%$ of the CPU is used.

The propagation process is illustrated in Figure 3. It starts from a source plane and ends when the destination plane is reached. 

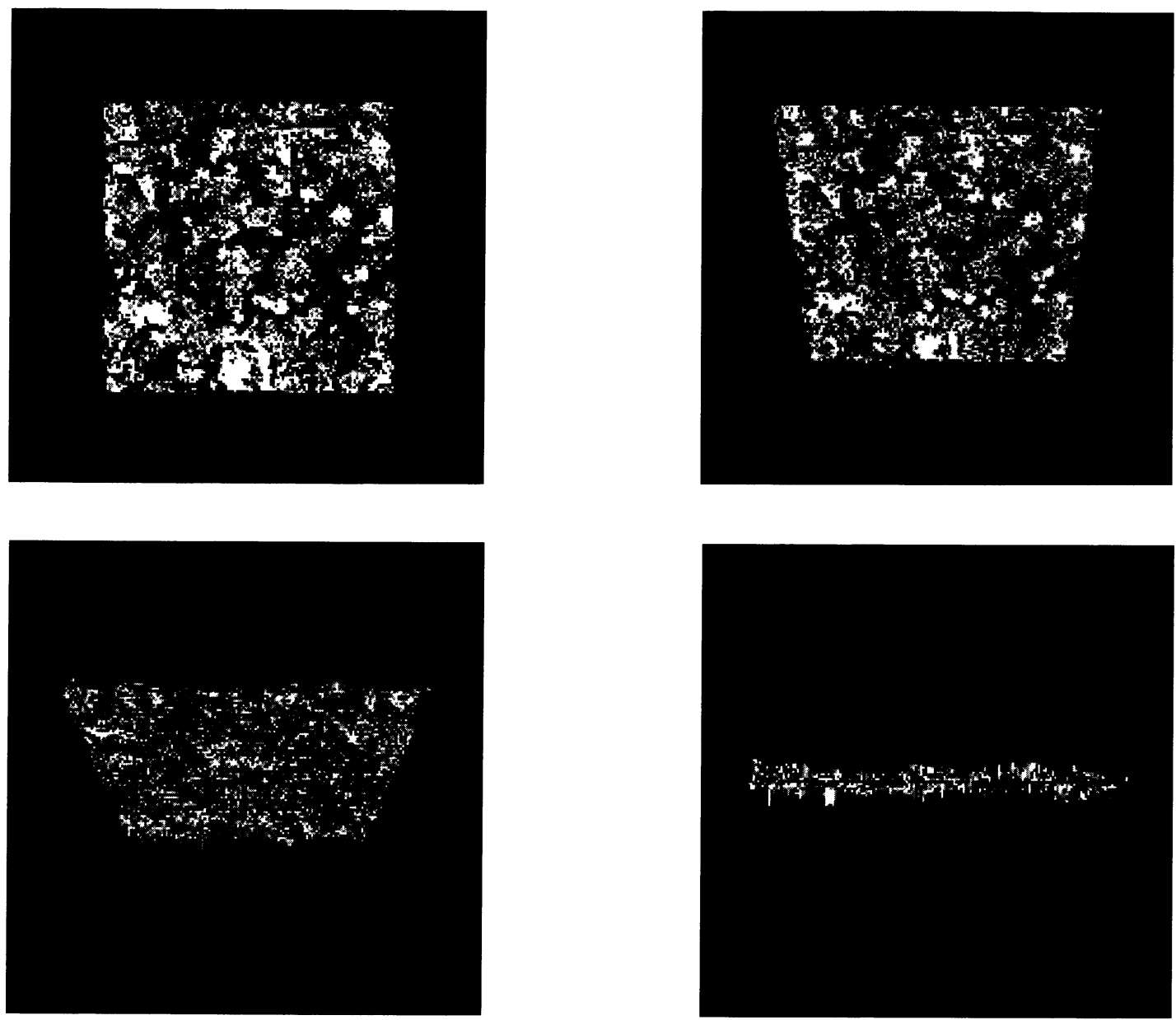

Fig. 6. - 3-D representations: passage from a front view to a cross-section of the pores of two joints in contact.

\section{Results}

Results are obtained under different forms. We first get general information about the porous medium: volume fraction of all the pores and of the pores reached by the propagation, and a path description: length of shortest paths (infinite for water proof samples), distributions of path lengths ... These give information about the pore structure. For example, a minimum path equal to the image width leads to the conclusion that the sample contains a "straight" path in the medium. The longer the paths are, the more winding they are. All these parameters are obtained either on the whole volume or on the destination plane only. Shortest path histograms (Fig. 4) illustrate the spectrum of path winding in the pores. The first histogram (Fig. 4a) presents the features of a very porous sample: an increasing front and a slow decrease. The second histogram (Fig. 4b) represents a less porous medium: several groups of paths can be distinguished, corresponding to different channels. The shortest path has a length of 505 pixels (less than the original image width), because of the bringing into contact which reduces the image intersection to a width of 505 pixels.

One also has access to a path cartography on the destination plane, which gives information on the path shape and location on that plane. Are they thin and winding or thick and direct? 
In Figure 5, a cartography and the corresponding histogram are associated. This histogram was thresholded to keep only the most important peaks (see Fig. 4b). The cartography presents in different gray levels the unreached pores (white), the reached pores belonging to the most numerous population of paths (dark gray) and the rest of the pores (light gray). The joint contact is also presented in three dimensions as it is illustrated in Figure 6.

We proceed the propagation on several samples with different levels of polishing, and this leads us to a percolation threshold from which the samples are waterproof or not. This can be useful to optimize the roughness of tap joints.

\section{Conclusion}

Rough surface porosity was studied through confocal microscopy and 3-D image processing. The acquisition process was briefly described, as well as two techniques of drift correction, according to the sample level of polishing: the first one by plane estimation and the second one involving a mathematical morphological operator, the closing by reconstruction. Then using image processing, the contact between two different samples was simulated. Finally, we used a propagation algorithm which presents two advantages compared to existing algorithms. First it works with 3-D images and second it is really efficient because of hierarchical queues. The various results obtained, (minimal path and time, path histograms, volume fraction, 2D cartography, 3-D representation) give useful information on the shape and structure of the propagation paths and on the interconnected pores in the medium.

\section{Acknowledgements}

The authors are indebted to Dr. G. Bernard-Granger (Ceramiques Techniques Desmarquest) for his help in the experimental part of the study.

\section{References}

[1] Brémond R., Jeulin D., Gateau P., Jarrin J. and Serpe G., Estimation of the transport properties of polymer composites by geodesic propagation. Microscopy 176 (1994) 167-177.

[2] Conan V., Gesbert S., Howard C.V., Jeulin D. and Meyer F., Geostatistical and morphological methods applied to three-dimensionnal microscopy, Microscopy 166 (1992) 169-184.

[3] Demarty C.H., Grillon F. and Jeulin D., Taux de portance - rugosité des surfaces des disques de robinetterie. Confidential N-14/96/MM, Centre de Morphologie Mathématique (École des Mines de Paris, April 1996).

[4] Gesbert S., Howard V., Jeulin D. and Meyer F., The use of basic morphological operations for 3d biological image analysis, Trans. Roy. Micros. Soc., MICRO 90 (july 1990) pp. 293-296.

[5] Gratin C. and Meyer F., Morphological three-dimensionnal analysis, Scanning Microsc. Suppl. (1992) 129-135.

[6] Jeulin D., Damage simulation in heterogenous materials from geodesic propagations, Eng. Computations 10 (1993) 81-91.

[7] Jeulin D. and Kurdy M.B., Quelques paramètres géodésiques pour caractériser la connexité des milieux biphasés, Technical Report N-24/89/MM, Centre de Morphologie Mathématique (École des Mines de Paris, November 1989).

[8] Jeulin D., Vincent L. and Serpe G., Propagation algorithms on graphs for physical application. Visual Commun. and Image Representation 3 (1992) 161-181.

[9] Jouannot P., Jernot J.P. and Guyon E., Étude de la connexité de structures aléatoires, C. R. Acad. Sci. Série IIb 321 (1995) 425-430.

[10] Meyer F., Mathematical morphology: from 2d to 3d, Microscopy 165 (1992) 5-28.

[11] Schmitt M. and Mattioli J., Morphologie Mathématique (Masson, Paris, 1994).

[12] Serra J., Image Analysis and Mathematical Morphology (Academic Press, London, 1982). 